\title{
Incertidumbre clínica durante la prescripción de proteína al paciente en estado crítico de Latinoamérica
}

\author{
The Clinical Uncertainty During Protein Prescription in Critically III Latin \\ American Patients
}

\author{
Luis Alejandro Ortiz-Reyes', Daren Heylan ${ }^{1,2 *}$ \\ Recibido: 18 de enero de 2019. Aceptado para publicación: 15 de marzo de 2019 \\ Publicado en línea, marzo 23 de 2019 \\ https://doi.org/10.35454/rncm.v2n1.058
}

\begin{abstract}
Resumen
Las Guías de Práctica Clínica recomiendan una dosis proteica en pacientes en estado crítico de 1,2 a 2,0 g/ $\mathrm{kg} /$ día. A pesar de esta recomendación, la cantidad real oscila entre 0,5 y $3,8 \mathrm{~g} / \mathrm{kg} /$ día, por lo cual existe en el mundo una controversia significativa acerca de la cantidad de proteína prescrita y la administrada. Esta revisión introduce el concepto de "equiponderación" clínica, balance o equilibrio en las estrategias de dosificación óptima de proteína. Además, se resalta la contribución limitada de pacientes de Latinoamérica (LATAM) en los Ensayos Clínicos Aleatorizados (ECA) existentes y cuestiona la generalización o extrapolación de sus resultados. Este escrito muestra la evidencia a favor y en contra de la prescripción de proteína en dosis altas e introduce el estudio Efecto de una Dosis Alta de Proteína en Pacientes Críticos (EFFORT) el cual intenta dar respuesta a la pregunta: ¿cuál es la mejor dosis de proteína para este tipo de pacientes?
\end{abstract}

Palabras clave: EFFORT, cuidados críticos, dosis de proteína, soporte nutricional, ensayos clínicos basados en registros.

\begin{abstract}
The guidelines recommendation for protein dose in critically ill patients is $1.2-2.0 \mathrm{~g} / \mathrm{kg} / \mathrm{d}$. Despite this recommendation, the actual amount delivered ranks between 0.5 and $3.8 \mathrm{~g} / \mathrm{kg} / \mathrm{d}$ therefore there is significant controversy in the amount of protein prescribed and delivered worldwide. This review approach the clinical equipoise, or a state of genuine uncertainty about two (dosing) strategies for protein dose. In addition, highlight the limited contribution of Latin American (LATAM) patients in published Randomized Clinical Trials (ECAs) questioning the generalizability of their results. This manuscript outline evidence for and against high protein dose and introduce the EFFORT trial which aims to answer the important question of what protein dose is the best for what type of patient?
\end{abstract}

Keywords: EFFORT; Critical care; Protein dose; Nutritional support; Clinical trials bases on records.

\section{INTRODUCCIÓN}

Una pregunta crítica para la comunidad dedicada a la nutrición en Unidades de Cuidados Intensivos (UCI) es: ¿una dosis alta de proteína tiene mejores resultados clínicos, cuando se compara con un aporte bajo de proteína? $?^{(1)}$.

Desde 2016, las guías de Soporte Nutricional Clínico en Cuidados Intensivos recomiendan aportes de pro-

Clinical Evaluation Research Unit, Department of Critical Care Medicine, Kingston General Hospital, Kingston, Ontario, Canada. teína en un rango amplio, entre 1,2 y $2,0 \mathrm{~g} / \mathrm{kg} /$ día, o aún mayor en pacientes quemados o con trauma ${ }^{(2)}$. A pesar de esta recomendación, en general, la cantidad real de proteína administrada oscila entre 0,5 y $3,8 \mathrm{~g} /$ $\mathrm{kg} /$ día (1,3 g/kg/día en promedio), según la Encuesta Internacional de Nutrición (INS, por su sigla en inglés) que ha recolectado $(\mathrm{n}=21.000)$ datos de 2007 a 2014 en las Unidades de Cuidado Intensivo del mundo ${ }^{(3)}$. Es

Profesor del Departamento de Ciencias de la Salud Pública, Queen's University, Kingston, Ontario, Canada.

*Correspondencia: Daren K. Heyland

Dkh2@queensu.ca 
posible que la administración de este rango tan amplio de proteína en la práctica clínica se deba a la debilidad del grado de recomendación de las guías clínicas. Es importante destacar, que ninguno de los ECA (Estudio Controlado Aleatorizado) de intervención nutricional reclutó pacientes de Latinoamérica ${ }^{(4-6)}$. La INS, es un estudio observacional, de gran escala, multicéntrico y multinacional que desde la década pasada describe, cada 12 a 18 meses, prácticas nutricionales locales.

El propósito principal de dicho proyecto fue definir el cuidado nutricional de alta calidad ${ }^{(7)}$. Un objetivo secundario exploró las relaciones entre prácticas nutricionales y resultados clínicos. Un análisis reciente demostró que alcanzar los requerimientos proteicos parece ser más importante que lograr las metas calóricas. $\mathrm{Al}$ analizar la ingesta calórica, se observó una reducción significativa asociada a la mortalidad a los 60 días, cuando se administra más de $80 \%$ de los requerimientos proteicos que cuando se provee menos de 80 \% (Razón de Momios [RM] 0,68 y 95 \% Intervalo de Confianza [IC]: 0,50 a 0,91) ${ }^{(8)}$.

En contraste, al analizar la ingesta proteica no se observó incremento en el efecto del aumento de proteínas en la administración de calorías (RM 0,89; $95 \%$ IC 0,71 a 1,12). Aunque la inferencia de este modelo estadístico es débil, es consistente con otros estudios observacionales que muestran una asociación entre optimización de proteína y supervivencia, pero ningún efecto en la ingesta de calorías ${ }^{(9,10)}$.

La INS contiene una pequeña fracción de pacientes latinoamericanos en estado crítico (1.500 de 21.139 [7 \%]). ¿Cómo discernir que los resultados de estos estudios multinacionales de nutrición se puedan generalizar o extrapolar a la práctica en LATAM? Vallejo y colaboradores reportaron que $74 \%(n=745 / 1053)$ de estos pacientes presentaban malnutrición de moderada a severa según la Evaluación Global Subjetiva ${ }^{(11)}$. Esta proporción es significativamente alta comparada con la observada (30 \% - 50 \%) en estudios Norteamericanos y Europeos ${ }^{(12-14)}$. Dado que pacientes con deficiencias nutricionales preexistentes se pueden beneficiar más del apoyo nutricional artificial ${ }^{(15)}$, la literatura actual impide extender las recomendaciones actuales de prescripción de proteína al contexto latinoamericano debido a la falta de datos disponibles resultado de estudios procedentes de esta zona. Para resolver la pregunta ¿Cuál es la dosis óptima de proteína?, se desarrolló el estudio EFFORT (Efecto de una dosis alta de proteína en pacientes críticos ${ }^{(16)}$. EFFORT, por su sigla en inglés, es un gran ECA, multicéntrico, multinacional, pragmático, realizado por voluntarios, basado en registros de 4.000 pacientes en alto riesgo nutricional; incluye pacientes críticamente enfermos de todo el mundo, aún pacientes de Latinoamérica, con el fin de maximizar la generalización de sus resultados. Este estudio aleatoriza los pacientes a una dosis alta de proteína $(\geq 2,2 \mathrm{~g} / \mathrm{kg} /$ día $)$ o tratamiento estándar $(\leq 1,2$ $\mathrm{g} / \mathrm{kg} /$ día) sin modificar la prescripción de calorías ${ }^{(16)}$. El principio de "equiponderación" clínica o balance (clinical equipoise) con respecto a la dosis óptima de proteína en cuidado crítico es parte fundamental del EFFORT. Este principio estipula que un ECA es únicamente ético en la medida en que exista, desde el inicio del estudio, un estado de genuina incertidumbre entre expertos sobre los efectos terapéuticos de cada brazo de tratamiento del estudio. El principio de "equiponderación” es respetado cuando el clínico carece de bases adecuadas para elegir entre dos o más opciones de tratamiento $^{(17)}$. El propósito de esta revisión es resumir la evidencia disponible que soporta la incertidumbre clínica sobre cuál es la prescripción correcta de proteína para pacientes críticamente enfermos.

\section{RACIONALIDAD PARA UNA DOSIS ALTA DE PROTEÍNA EN CUIDADO CRÍTICO}

La mayoría de estudios básicos que evalúan los efectos metabólicos de la administración intravenosa de aminoácidos (AA) sustentan la afirmación de que la infusión de AA estimula la síntesis proteica de novo, resultando en un mayor balance proteico corporal ${ }^{(17)}$. Además, estudios han mostrado que una dosis alta resulta en un efecto anabólico con un balance nitrogenado positivo ${ }^{(18,19)}$. Roselli y colaboradores en un ECA compararon la administración de nutrición enteral hipocalórica, hiperproteica $(\mathrm{n}=40)$ frente a una nutrición enteral isocalórica $(n=40)$, el grupo de intervención recibió más proteína en forma significativa $(1,4$ vs $0,76 \mathrm{~g} / \mathrm{kg}, \mathrm{p} \leq 0,0001)$, con una mejora en el puntaje SOFA a las 48 horas y menos episodios hiperglicémicos. Sin embargo, no existieron diferencias en otros resultados clínicos importantes. Además, los autores reconocieron que el pequeño tamaño de muestra y la ausencia de poder del estudio son las principales limitaciones ${ }^{(20)}$. Ferrie y colaboradores aleatorizaron pacientes médicos/quirúrgicos de la UCI en dos grupos, un grupo de pacientes recibió infusión de AA estándar $(0,8 \mathrm{~g} / \mathrm{kg} /$ día $)$ y el otro, infusión de dosis alta de AA $(1,2 \mathrm{~g} / \mathrm{kg} /$ día $)$. El grupo de dosis alta $(\mathrm{n}=59)$ tuvo un aumento significativo de la masa muscular con 
tendencia al aumento de la fuerza de agarre de la mano en comparación con el grupo estándar $(\mathrm{n}=60)^{(21)}$. No obstante, la cantidad de proteína recibida en ambos grupos fue marginal $(0,9 v s 1,1 \mathrm{~g} / \mathrm{kg} /$ día $)$, pero ¿cómo una diferencia tan pequeña en la ingesta se puede traducir en grandes cambios en la masa muscular y en la fuerza? Esto crea controversia.

Resultados de una base de datos con más de 7.000 pacientes críticamente enfermos siguiere que 30 gramos de proteína o 1.000 calorías/ día adicionales durante los primeros 12 días en la UCI se asocia a una reducción de complicaciones infecciosas, días dependientes de la ventilación mecánica, mejoramiento en la recuperación física a corto plazo y supervivencia ${ }^{(15}$, 22-24). Nicolo y colaboradores observaron, en un análisis de una base de datos $(\mathrm{n}=2,828)$, una asociación entre la administración $>80 \%$ de los requerimientos de proteína con una reducción en la mortalidad a los 60 días (RM 0,68; $95 \%$ IC 0,50 a 0,91) pero este efecto no se observó cuando se alcanzaba $>80 \%$ de los requerimientos calóricos (RM 0,92; 95 \% IC 0,65 a 1,30$)^{(8)}$. Si bien la inferencia de estos estudios observacionales son débiles en comparación con la de los ECA, estas observaciones son consistentes con otros estudios observacionales que muestran una asociación entre la ingesta proteica óptima y la supervivencia, pero ningún efecto o efecto negativo en la ingesta calórica. Hoffer y Bistrian sugieren que una ingesta proteica de hasta 2,5 $\mathrm{g} / \mathrm{kg} /$ día es segura en pacientes con lesión renal aguda en diálisis, excepto, quizá, en pacientes con hipotensión refractaria (lo cual causa hipoperfusión hepática) y enfermedades hepáticas severas. Se reconoce que una alta dosis de proteína puede causar niveles altos de urea, pero el riesgo o los daños asociados a estos resultados se desconocen ${ }^{(25)}$.

\section{RACIONALIDAD PARA OBJETAR UNA DOSIS ALTA DE PROTEÍNA EN CUIDADO CRÍTICO}

El estudio multicéntrico de fase II Nephro-Protective de Doig y colaboradores evaluó en 474 pacientes el efecto fisiológico sobre la función renal de la administración intravenosa de AA. En el estudio se comparó la administración de aminoácidos intravenosos (AA IV) a una dosis de hasta $2,0 \mathrm{~g} / \mathrm{kg} /$ día (dosis alta) contra la práctica estándar. Estos resultados son la evidencia más fuerte en contra de una dosis alta de proteína ${ }^{(26)}$. La duración de la disfunción renal y otros resultados clínicos no fueron diferentes entre grupos y sugieren que una dosis proteica de hasta $2 \mathrm{~g} / \mathrm{kg} /$ día no mejora los resultados en grupos heterogéneos, ya que dos pacientes en estado crítico no se benefician de la ingesta proteica de igual manera. Dos estudios observacionales (n $=63 \mathrm{y} \mathrm{n}=455)$ reportaron efectos adversos asociados a una alta ingesta proteica, demostrando que el aumento de la ingesta proteica (media $0,67 \mathrm{~g} / \mathrm{kg} /$ día) se asoció a aumento de la pérdida muscular ${ }^{(27)}$. También encontraron que la administración de proteína $>0,8 \mathrm{~g} / \mathrm{kg} /$ día antes del tercer día se asoció a mayor mortalidad en comparación con la misma ingesta provista después ${ }^{(28)}$. Además, un análisis post-hoc del estudio EPaNIC $(\mathrm{n}=4.640)$, demostró que una ingesta hiperproteica durante los primeros 3 días se asocia a menor probabilidad de egreso temprano de la UCI ${ }^{(29)}$.

Un análisis post-hoc de un ECA $(\mathrm{n}=66)$ indicó que una ingesta hiperproteica durante la primera semana se asoció a una reducción significativa de la supervivencia, pero la proteína provista después de la primera semana parecía tener un efecto protector ${ }^{(30)}$. Estas observaciones sugieren daño potencial asociado a un aumento de la ingesta proteica, particularmente en la fase aguda de la enfermedad, pero estas deben ser consideradas observaciones generadoras de hipótesis. Sin embargo, estos resultados contribuyen a la incertidumbre sobre el rol de la ingesta de proteína en pacientes en estado crítico. Estudios preclínicos y clínicos sugieren que la proteína/AA puede anular la autofagia, pero no puede reducir el catabolismo endógeno en cuidado crítico $^{(31)}$. Las implicaciones clínicas de estos resultados continúan siendo indeterminadas. En resumen, la evidencia actual (estudios con diseños limitados, tamaño inadecuado de la muestra, reclutamiento heterogéneo de pacientes, resultados significativos pero controversiales, donde la dosis de proteína no fue la principal intervención, y una muestra no representativa de LATAM) aumenta la incertidumbre acerca de ¿qué dosis de proteína es benéfica según este tipo de pacientes?

\section{RESULTADOS CLÍNICOS EN FALLA RENAL}

Investigadores sugieren que la enfermedad renal aguda (ERA) ocurre en más de $50 \%$ de los pacientes en la UCI y $15 \%$ requiere terapia de reemplazo renal (TRR) ${ }^{(32)}$. Un balance nitrogenado no positivo está presente en la ERA y la terapia de reemplazo renal. La ERA compromete diversas funciones homeostáticas renales, incluyendo mantenimiento del balance ácido-base, alteración de la conversión de AA, y la TRR resulta en pérdida de $\mathrm{AA}^{(33)}$. Aunque la ERA es común durante la enfermedad crítica, los reportes de esta enfermedad no son consisten- 
tes, algunos informan una mediana de creatinina entre ambos grupos y otros reportan porcentaje de pacientes con ERA, limitando la inferencia para la dosis óptima de proteína en la enfermedad renal aguda.

Como se ha indicado, el estudio Nephro-Protective presenta la evidencia más consistente en contra de dosis alta de proteína en pacientes en estado crítico ${ }^{(26)}$. La racionalidad detrás de este ECA se basa en modelos animales que han demostrado el aumento del flujo sanguíneo renal en respuesta a una infusión corta de AA y en que el aumento del flujo sanguíneo puede proteger el riñón de lesiones isquémicas; se basa también en diversos estudios que documentaron mejoramiento del balance nitrogenado en pacientes en diálisis con ingesta alta de $\mathrm{AA}^{(34-38)}$. Un análisis de subgrupos de un ECA con 27 UCI que evalúa guías nutricionales identificó 242 pacientes en estado crítico con alto riesgo de disfunción renal durante la admisión al estudio y encontró una menor probabilidad de requerir TRR en aquellos con dosis altas de proteína ${ }^{(39-40)}$. Un ECA $(n=53)$ en pacientes en estado crítico demostró que una infusión a corto plazo de AA intravenosos resulta en un mejoramiento acelerado de la ERA severa, particularmente en aquellos con insuficiencia renal oligúrica, recibieron diálisis, y en aquellos que desarrollaron sepsis ${ }^{(41)}$. Otro ECA $(n=14)$ comparó 2 dosis de AA IV en pacientes en estado crítico sin insuficiencia renal oligúrica (aclaramiento o depuración de creatinina $<50 \mathrm{~mL} / \mathrm{min}$ ), y en aquellos que recibieron una dosis alta de AA tuvieron mayor probabilidad de preservar el efecto de la diuresis y requerir menor furosemida para alcanzar un balance negativo ${ }^{(42)}$. Finalmente, un análisis post hoc del estudio Nephro-Protective sugiere un mejoramiento en la supervivencia en pacientes con función renal normal recibiendo AA intravenosos (21/179 [11,7 \%]) comparado con el cuidado estándar (37/189 [19,6\%]), pero también sugieren menor supervivencia en aquellos con insuficiencia renal basal $(17 / 60$ [28,3\%] vs $7 / 46[15,2 \%])^{(43)}$. La observación anterior no fue significativa en análisis ajustado por covariables $(-0,6 \%$; $95 \% \mathrm{CI},-16,2$ a 15,2; $\mathrm{p}=0,95)$. Sin embargo, se encontraron diversas limitaciones en este análisis. Primero, el número de subgrupos es pequeño y la tasa de eventos baja, así los resultados son inestables y frágiles; Segundo, existe inconsistencia entre los resultados del estudio (solo mortalidad mostró diferencias significativas entre grupos; pero la calidad de vida y función física tuvieron una tendencia a empeorar con el tratamiento); Tercero, dado la racionalidad subyacente del estudio Nepro-Protective, que los AA intravenosos fue- ron empleados para mejorar los resultados en pacientes con insuficiencia renal, existe falta de plausibilidad biológica para este resultado de subgrupos. No obstante, queda planteada la hipótesis de que la suplementación intravenosa de AA podría aumentar el daño a pacientes con falla renal.

\section{EL CAMINO A SEGUIR}

El área de nutrición en cuidado crítico permanece inmóvil en un estado de incertidumbre clínica para la dosis óptima de proteína. Existe información que apoya tanto el beneficio como el peligro de la dosis de proteína que se administra en la actualidad, sugiriendo que: 1) una dosis de proteína alta es mejor que una dosis baja; 2) una dosis baja es mejor que una dosis alta; 3) una dosis baja no es mejor ni peor que una dosis alta. A pesar de esto, notamos que la mayoría de la evidencia surge de ECA unicéntricos, estudios observacionales, o análisis post hoc. Además, la mayoría de los estudios fueron realizados en países no latinoamericanos; así, las hipótesis de los resultados no se pueden extrapolar a todos los pacientes en estado crítico. Postulamos que no existen bases adecuadas para elegir entre dos opciones de proteína disponibles para los clínicos, por lo tanto, se sugiere realizar estudios basados en el principio de "equiponderación" clínica.

Las diversas fuentes de información que contribuyen a conocer la dosis óptima de proteína requerida en cuidado crítico no fueron diseñadas en forma rigurosa. Los clínicos deben ser precavidos al sobre interpretar ECA unicéntricos, estudios observacionales, análisis post hoc, opiniones de expertos, estudios donde la dosis de proteína no fue la intervención, y en aquellos con resultados clínicos débiles. Todos los estudios incluyen poblaciones heterogéneas de pacientes en estado crítico y muestras no representativas para Latinoamérica, situación que debería estimular a los colegas latinoamericanos dedicados a la nutrición en cuidado crítico a participar en el EFFORT. Es claro que no existe evidencia definitiva de grandes ECA, multinacionales, multicéntricos, que evalúan los diferentes niveles de ingesta proteica en pacientes con alto riesgo nutricional de diversas regiones del mundo.

El estudio EFFORT es un ECA pragmático, multinacional, multicéntrico, basado en registros, que incluye países de Latinoamérica ${ }^{(16)}$. El objetivo es reclutar pacientes adultos críticos admitidos a la UCI que requieren ventilación mecánica con una duración total actual o esperada de más de 48 horas desde el cribado que tengan 
uno o más de los siguientes factores de alto riesgo nutricional: Índice de Masa Corporal $<250 \geq 35$; malnutrición de moderada a severa definido por las evaluaciones locales, fragilidad (escala de fragilidad clínica $\geq 5$, sarcopenia (SARC-F $\geq 4$ ), ventilación mecánica esperada $\geq 96$ horas desde el cribado. Además, se han planeado, a priori, diversos subgrupos de análisis de diferentes subpoblaciones (falla renal o aquellos en TRR, cirugías masivas, trauma múltiple, y quemados graves) que se espera que se beneficien de alta dosis de proteína. Por lo tanto, se confía en que estos pacientes sean inscritos al estudio (en lugar de ser excluidos porque se crea que una dosis, mayor o menor, de proteína es mejor) permitiendo explorar a priori el efecto de alta contra baja dosis en estos subgrupos. El tamaño de muestra es grande ( $\mathrm{n}=$ 4.000) suficiente para detectar un efecto pequeño del tratamiento. Con la estrategia de dosis usadas, el estudio EFFORT está diseñado para crear una adecuada separación de la ingesta proteica en ambos grupos y así evitar las críticas de estudios anteriores.

El estudio EFFORT, a excepción de la dosificación de proteína, no pretende modificar la práctica usual del clínico. Se espera que se provea la proteína vía enteral a través de fórmulas enterales y suplementos de proteína modular disponibles en sus instituciones o AA vía parenteral o ambos según su práctica clínica.

Para alcanzar el objetivo proteico, se recalca la importancia de evitar la sobrealimentación calórica. Para pacientes no obesos, se sugiere una prescripción calórica de 20 - $25 \mathrm{kcal} / \mathrm{kg} /$ día, usando una fórmula simple basada en el peso. Para pacientes obesos, se utilizan las guías de A.S.P.E.N. y S.C.C.M. que indican que si la calorimetría indirecta es usada, el objetivo calórico no debe exceder $65 \%$ - $70 \%$ de los requerimientos medidos $^{(2,44)}$. Si la calorimetría indirecta no está disponible o no se usa, sugerimos usar ecuaciones basadas en el peso, $11-14 \mathrm{kcal} / \mathrm{kg}$ actual peso corporal por día para pacientes con IMC entre 30 - 50, y 22 $25 \mathrm{kcal} / \mathrm{kg}$ peso ideal corporal por día para pacientes con IMC > 50. La ingesta de energía de suplementos proteicos modulares, y dosis de propofol serán incluidas en estos objetivos. Dado que el factor tiempo en la administración de proteína es un aspecto sensible, en el EFFORT la intervención del estudio comenzará tan pronto se haga la aleatorización y terminará al alta de la UCI, hospital, transición a vía oral, o muerte, lo que ocurra primero. Los resultados del estudio son mortalidad hospitalaria a los 60 días, tiempo de ser dado de alta vivo del hospital, mortalidad hospitalaria, readmisión a la UCI y hospital, duración de la ventilación mecá- nica, estancia en la UCI y hospitalaria, y adecuación nutricional. El estudio EFFORT está bajo el análisis de intención a tratar; y por lo tanto, todos los pacientes aleatorizados serán incluidos en el análisis.

\section{CONCLUSIONES}

Existe controversia considerable a cerca de la dosis de proteína que se debería prescribir a los pacientes en estado crítico, motivada en parte por la información disponible en las guías de práctica clínica publicadas recientemente (que recomiendan iniciar nutrición enteral temprana y prescribir de 1,2 a $2,0 \mathrm{~g} / \mathrm{kg} /$ día o más). Este artículo resume evidencia, en contra y a favor de una dosis alta de proteína, producto en su mayoría de ECA unicéntricos, estudios observacionales o análisis post-hoc. Claramente, se necesitan grandes ECA, prospectivos, multicéntricos que evalúen las diferentes dosis de proteína en pacientes en estado crítico con alto riesgo nutricional y proporcionen evidencia definitiva. El estudio EFFORT ayudará a resolver esta controversia siempre y cuando los clínicos reconozcan que la incertidumbre enmarca la evidencia aplicada a su práctica clínica, e inscriban a sus pacientes, en especial latinoamericanos, lo que permitirá extrapolar y generalizar estos resultados a pacientes de LATAM. Con seguridad los resultados del EFFORT, aumentará la confianza en la dosis óptima de proteína que requieren los pacientes en estado crítico alrededor del mundo.

\section{Conflicto de intereses}

Daren K. Heyland ha recibido fondos de los Institutos Canadienses de Investigación en Salud e Institutos Nacionales de Investigación en Salud, así como honorarios de Nestlé Ciencias de la Salud. También ha recibido un salario como consultor y fondos de investigación por Lyric Pharmaceuticals, Emotion Medical y GlaxoSmithKline.

\section{Financiación}

El presente artículo no tuvo financiación.

\section{Referencias bibliográficas}

1. Arabi YM, Casaer MP, Chapman M, et al. The intensive care medicine research agenda in nutrition and metabolism. Intensive Care Med. 2017;43(9):1239-1256. doi:10.1007/ s00134-017-4711-6.

2. Taylor BE, McClave SA, Martindale RG, et al. Guidelines for the Provision and Assessment of Nutrition Support 
Therapy in the Adult Critically Ill Patient: Society of Critical Care Medicine (SCCM) and American Society for Parenteral and Enteral Nutrition (A.S.P.E.N.). Crit Care Med. 2016;44(2). http://journals.lww.com/ccmjournal/ Fulltext/2016/02000/Guidelines_for_the_Provision_and Assessment_of.20.aspx.

3. Heyland DK, Weijs PJM, Coss-Bu JA, et al. Protein Delivery in the Intensive Care Unit: Optimal or Suboptimal? Nutr Clin Pract. 2017;32(1 Suppl):58S-71S. doi: $10.1177 / 0884533617691245$.

4. Arabi YM, Aldawood AS, Haddad SH, et al. Permissive Underfeeding or Standard Enteral Feeding in Critically Ill Adults. N Engl J Med. 2015;372(25):2398-2408. doi:10.1056/NEJMoa1502826.

5. Rice TW, Wheeler AP, Thompson BT, et al. Initial Trophic vs Full Enteral Feeding in Patients With Acute Lung Injury: The EDEN Randomized Trial. JAMA J Am Med Assoc. 2012;307(8):795-803. doi:10.1001/jama.2012.137.

6. Casaer MP, Hermans G, Wilmer A, Van den Berghe G. Impact of early parenteral nutrition completing enteral nutrition in adult critically ill patients (EPaNIC trial): A study protocol and statistical analysis plan for a randomized controlled trial. Trials. 2011. doi:10.1186/1745-6215-12-21.

7. Cahill NE, Dhaliwal R, Day AG, Jiang X, Heyland DK. Nutrition therapy in the critical care setting: what is "best achievable" practice? An international multicenter observational study. Crit Care Med. 2010;38(2):395-401. doi:10.1097/ CCM.0b013e3181c0263d.

8. Nicolo M, Heyland DK, Chittams J, Sammarco T, Compher C. Clinical Outcomes Related to Protein Delivery in a Critically Ill Population: A Multicenter, Multinational Observation Study. JPEN J Parenter Enteral Nutr. 2016;40(1):45-51. doi:10.1177/0148607115583675.

9. Allingstrup MJ, Esmailzadeh N, Wilkens Knudsen A, et al. Provision of protein and energy in relation to measured requirements in intensive care patients. Clin Nutr. 2012. doi:10.1016/j.clnu.2011.12.006.

10. Weijs PJM, Looijaard WGPM, Beishuizen A, Girbes ARJ, Oudemans-van Straaten HM. Early high protein intake is associated with low mortality and energy overfeeding with high mortality in non-septic mechanically ventilated critically ill patients. Crit Care. 2014. doi:10.1186/s13054-014-0701-z.

11. Vallejo KP, Martínez CM, Matos Adames AA, et al. Current clinical nutrition practices in critically ill patients in Latin America: a multinational observational study. Crit Care. 2017;21(1):227. doi:10.1186/s13054-017-1805-z.

12. Barreto EF, Kanderi T, DiCecco SR, et al. Sarcopenia Index Is a Simple Objective Screening Tool for Malnutrition in the Critically Ill. JPEN J Parenter Enteral Nutr. 2018. doi:10.1002/jpen.1492.

13. Canales C, Elsayes A, Yeh DD, et al. Nutrition Risk in Critically Ill Versus the Nutritional Risk Screening 2002: Are They Comparable for Assessing Risk of Malnutrition in Critically
Ill Patients? JPEN J Parenter Enteral Nutr. 2018. doi:10.1002/ jpen.1181.

14. Verghese PP, Mathai AS, Abraham V, Kaur P. Assessment of malnutrition and enteral feeding practices in the critically ill: A single-centre observational study. Indian J Anaesth. 2018;62(1):29-35. doi:10.4103/ija.IJA_513_17.

15. Alberda C, Gramlich L, Jones $\mathrm{N}$, et al. The relationship between nutritional intake and clinical outcomes in critically ill patients: results of an international multicenter observational study. Intensive Care Med. 2009;35(10):1728-1737. doi:10.1007/s00134-009-1567-4.

16. Heyland DK, Patel J, Bear D, et al. The Effect of Higher Protein Dosing in Critically Ill Patients: A Multicenter Registry-Based Randomized Trial: The EFFORT Trial. JPEN J Parenter Enteral Nutr. 2018. doi:10.1002/jpen.1449.

17. Heyland DK, Stapleton R, Compher C. Should We Prescribe More Protein to Critically Ill Patients? Nutrients. 2018;10(4). doi: $10.3390 /$ nu10040462.

18. Liebau F, Sundstrom M, van Loon LJC, Wernerman J, Rooyackers O. Short-term amino acid infusion improves protein balance in critically ill patients. Crit Care. 2015;19:106. doi:10.1186/s13054-015-0844-6.

19. Dickerson RN, Pitts SL, Maish GO 3rd, et al. A reappraisal of nitrogen requirements for patients with critical illness and trauma. J Trauma Acute Care Surg. 2012;73(3):549-557. doi:10.1097/TA.0b013e318256de1b.

20. Rosselli D, Rugeles S-J, Rueda J-D, Díaz C-E. Hyperproteic hypocaloric enteral nutrition in the critically ill patient: A randomized controlled clinical trial. Indian J Crit Care Med. 2013. doi:10.4103/0972-5229.123438.

21. Ferrie S, Allman-Farinelli M. Commonly used "nutrition" indicators do not predict outcome in the critically ill: a systematic review. Nutr Clin Pract. 2013;28(4):463-484. doi: $10.1177 / 0884533613486297$.

22. Heyland DK, Cahill N, Day AG. Optimal amount of calories for critically ill patients: depends on how you slice the cake! Crit Care Med. 2011;39(12):2619-2626. doi:10.1097/ CCM.0b013e318226641d.

23. Heyland DK, Stephens KE, Day AG, McClave S a. The success of enteral nutrition and ICU-acquired infections: A multicenter observational study. Clin Nutr. 2011;30(2):148-155. doi:10.1016/j.clnu.2010.09.011.

24. Wei X, Day AG, Ouellette-Kuntz H, Heyland DK. The Association Between Nutritional Adequacy and Long-Term Outcomes in Critically Ill Patients Requiring Prolonged Mechanical Ventilation: A Multicenter Cohort Study. Crit Care Med. 2015;43(8):1569-1579. doi:10.1097/ CCM.0000000000001000.

25. Hoffer LJ, Bistrian BR. Why critically ill patients are protein deprived. JPEN J Parenter Enter Nutr. 2013;37. doi: $10.1177 / 0148607113478192$.

26. Doig GS, Simpson F, Bellomo R, et al. Intravenous amino acid therapy for kidney function in critically ill patients: a rando- 
mized controlled trial. Intensive Care Med. 2015;41(7):11971208. doi:10.1007/s00134-015-3827-9.

27. Puthucheary ZA, Rawal J, McPhail M, et al. Acute skeletal muscle wasting in critical illness. JAMA - J Am Med Assoc. 2013. doi:10.1001/jama.2013.278481.

28. Koekkoek WACK, van Setten CHC, Olthof LE, Kars JCNH, van Zanten ARH. Timing of PROTein INtake and clinical outcomes of adult critically ill patients on prolonged mechanical VENTilation: The PROTINVENT retrospective study. Clin Nutr. 2018. doi:10.1016/j.clnu.2018.02.012.

29. Casaer MP, Mesotten D, Hermans G, et al. Early versus late parenteral nutrition in critically ill adults. N Engl J Med. 2011;365. doi:10.1056/NEJMoa1102662.

30. Braunschweig CL, Freels S, Sheean PM, et al. Role of timing and dose of energy received in patients with acute lung injury on mortality in the Intensive Nutrition in Acute Lung Injury Trial (INTACT): a post hoc analysis. Am J Clin Nutr. 2017;105(2):411-416. doi:10.3945/ajcn.116.140764.

31. Gunst J, Vanhorebeek I, Thiessen SE, Van den Berghe G. Amino acid supplements in critically ill patients. Pharmacol Res. 2018;130:127-131. doi:10.1016/j.phrs.2017.12.007.

32. Gaudry S, Hajage D, Schortgen F, et al. Initiation Strategies for Renal-Replacement Therapy in the Intensive Care Unit. N Engl J Med. 2016;375(2):122-133. doi:10.1056/ NEJMoa1603017.

33. Btaiche IF, Mohammad RA, Alaniz C, Mueller BA. Amino Acid requirements in critically ill patients with acute kidney injury treated with continuous renal replacement therapy. Pharmacotherapy. 2008;28(5):600-613. doi:10.1592/ phco.28.5.600.

34. Roberts PR, Black KW, Zaloga GP. Enteral feeding improves outcome and protects against glycerol-induced acute renal failure in the rat. Am J Respir Crit Care Med. 1997;156(4 Pt 1):1265-1269. doi:10.1164/ajrccm.156.4.9607003.

35. Bellomo R, Seacombe J, Daskalakis M, et al. A prospective comparative study of moderate versus high protein intake for critically ill patients with acute renal failure. Ren Fail. 1997;19(1):111-120.

36. Bellomo R, Tan HK, Bhonagiri S, et al. High protein intake during continuous hemodiafiltration: impact on amino acids and nitrogen balance. Int J Artif Organs. 2002;25(4):261-268.
37. Scheinkestel CD, Adams F, Mahony L, et al. Impact of increasing parenteral protein loads on amino acid levels and balance in critically ill anuric patients on continuous renal replacement therapy. Nutrition. 2003;19(9):733-740.

38. Fiaccadori E, Maggiore U, Rotelli C, et al. Effects of different energy intakes on nitrogen balance in patients with acute renal failure: a pilot study. Nephrol Dial Transplant. 2005;20(9):1976-1980. doi:10.1093/ndt/gfh956.

39. Doig GS, Simpson F, Finfer S, et al. Effect of evidence-based feeding guidelines on mortality of critically ill adults: a cluster randomized controlled trial. JAMA. 2008;300. doi:10.1001/ jama.2008.826.

40. Doig GS, Simpson F, Sweetmant EA, Bellomo R. Improved Nutritional Support Is Associated with Reduced Renal Dysfunction in Critical Illness: A Post-Hoc Exploratory Subgroup Analysis. In: A41. CLINICAL TRIALS IN ICU. American Thoracic Society International Conference Abstracts. American Thoracic Society; 2009:A1567. doi:doi:10.1164/ajrccm-conference.2009.179.1_ MeetingAbstracts.A1567.

41. Abel RM, Beck CHJ, Abbott WM, Ryan JAJ, Barnett GO, Fischer JE. Improved survival from acute renal failure after treatment with intravenous essential L-amino acids and glucose. Results of a prospective, double-blind study. N Engl J Med. 1973;288(14):695-699. doi:10.1056/ NEJM197304052881401.

42. Singer P. High-dose amino acid infusion preserves diuresis and improves nitrogen balance in non-oliguric acute renal failure. Wien Klin Wochenschr. 2007;119(7-8):218-222. doi:10.1007/s00508-007-0794-3.

43. Zhu R, Allingstrup MJ, Perner A, Doig GS, NephroProtective Trial Investigators Group. The Effect of IV Amino Acid Supplementation on Mortality in ICU Patients May Be Dependent on Kidney Function: Post Hoc Subgroup Analyses of a Multicenter Randomized Trial. Crit Care Med. 2018;46(8):1293-1301. doi:10.1097/ CCM.0000000000003221.

44. Mueller C, Compher C, Ellen DM, Directors the AS for $\mathrm{P}$ and EN (A. SPEN. B of. A.S.P.E.N. Clinical Guidelines: Nutrition Screening, Assessment, and Intervention in Adults. J Parenter Enter Nutr . 2011;35(1):16-24. doi: $10.1177 / 0148607110389335$. 